\title{
UNA MANERA DIFERENTE DE APRENDER INVESTIGANDO EN LAS ESCUELAS SECUNDARIAS DEL PERU
}

\section{A DIFFERENT WAY TO LEARN INVESTIGATING IN THE HIGH SCHOOLS OF THE PERU}

\author{
David Esteban Espinoza ${ }^{1}$
}

\section{RESUMEN}

La experiencia del autor y sus alumnos está centrada en la elaboración de 14 experimentos relacionados al estudio de la electricidad; sus efectos, aplicaciones y relación con el magnetismo, tratando de aprender investigando en el curso de física de manera extraescolar. Se utilizó en gran parte materiales reciclados. Para que las demostraciones puedan ser más didácticas se decidió manipular las experiencias a través de la computadora, se elaboró un programa muy sencillo en BORLAND C++. El equipo se puede trasladar fácilmente con su maletín portátil cuando se requiere utilizar en otro lugar. El trabajo final fue denominado "Demostrador Múltiple Experimental de Electricidad y Magnetismo", al ser expuesta dentro del marco de las Actividades Científicas Extraescolares a nivel nacional e internacional obtuvo premios importantes. Posteriormente los escolares emprendiendo una capacidad de iniciativa, liderazgo y tratando de continuar e irradiar su experiencia se organizaron alrededor del club de ciencias "Incubadora de Investigadores Científicos Escolares" para realizar trabajos de investigación científica y tecnológica; periódica, despertando el interés por aprender investigando de otros escolares que los precederán, son asesorados por maestros e investigadores entusiastas.

Palabras clave: Diferente, aprender, investigando, escuelas secundarias, Perú.

\section{ABSTRACT}

The author's experience and their students are centered in the elaboration of 14 experiments related to the study of the electricity; their effects, applications and relationship with the magnetism, trying to learn investigating in the course of physics in way extra scholar. It was largely used recycled materials. So that the demonstrations can be but didactic he/ she decided to manipulate the experiences through the computer, a very simple program was elaborated in BORLAND $\mathrm{C}++$. The team can move easily with its portable handbag when it is required to use in another place. The final work was denominated "Experimental Multiple Demonstrator of Electricity and Magnetism", when being exposed inside the mark of the Scientific Activities Extra scholars at national and international level he/she obtained important prizes. Later on the scholars undertaking an initiative capacity, leadership and trying to continue and to irradiate their experience was organized around the club of sciences "Incubator of School Scientific Investigators" to carry out works of scientific and technological investigation; periodic, waking up the interest to learn investigating of other scholars that will precede them, is advised by teachers and enthusiastic investigators.

Key words: Different, to learn, investigating, high schools, Peru.

\section{INTRODUCCION}

Realizamos el trabajo con la intención de sensibilizar a la comunidad educativa sobre la problemática de la enseñanza de la física en el nivel secundario, ejercitar la creatividad e innovación desde la escuela. Entre los antecedentes tenemos la experiencia de la Universidad Peruana Cayetano Heredia en la que sus estudiantes desde los primeros años realizan trabajos de investigación; "Modulo Múltiple Experimental de Física" trabajo presentado en la I FERIA CIENTIFICA Y TECNOLOGICA UNIVERSITARIA por David Esteban Espinoza el año 2000; tenemos también referencias publicitarias de las empresas LEYBOLD y PASCO las cuales ofrecen equipos de experimentación física manipuladas desde la computado- ra.

El problema se generó por lo difícil e irrelevante que es enseñar y aprender física en forma teórica, por las deficiencias de la curricula, falta de laboratorios adecuados, horarios rígidos de clase que tiene como consecuencia el bajo rendimiento académico y deficiente comprensión de los cursos de ciencias por los alumnos el que se ve reflejado a través de sus notas, el interés por averiguar como funcionan las cosas, la capacidad de análisis, curiosidad por averiguar que hay detrás de cada innovación tecnológica se ha perdido. Se realizó una recopilación de información socio económica de los alumnos al momento de iniciar nuestra investigación: se tienen los siguientes casos de problemática escolar: $5,34 \%$ de agresiones físicas; $1,86 \%$ de drogadicción;
$0,37 \%$ de alcoholismo reportados entre los alumnos; la mayoría de ellos poseen escasos recursos económicos, se detectaron interés por las pandillas juveniles desinterés por los cursos de ciencias básicas, aburrimiento, cansancio, no es raro encontrar alumnos que huyen del colegio, que desean que el profesor no desarrolle clases. Los adolescentes entrevistados solicitan un modo diferente de aprender donde puedan explayar sus potencialidades e intereses.

El objetivo general fue diseñar, construir evaluar empleando materiales reciclables, un conjunto sistematizado de materiales didácticos manipulados desde la computadora, con los cuales se pueda efectuar una amplia gama de experimentos en electricidad y

\footnotetext{
${ }^{1}$ Centro de Estudios e Investigación de la Educación Peruana. Profesor de física - matemática colegio "San Luis".
} 
magnetismo haciendo más comprensible la física e incrementando nuestro interés por ella. El objetivo específico: Brindar a los escolares la oportunidad de desarrollar su creatividad, curiosidad natural e iniciarlos en la experiencia de aprender investigando, construyendo y hasta en algunos casos divirtiéndose. La hipótesis de trabajo fue, en colegios estatales como el nuestro las deficiencias del sistema educativo, inadecuada curricula, falta de material didáctico genera que se enseñe y aprenda física en forma teórica priorizando la "tiza, pizarra, memoria" por lo que iniciarlos en la investigación permitiría aprender de un modo diferente el tema elegido, facilitaría la utilización de materiales domésticos en desuso, reciclables dentro de una cultura ecológica para construir módulos de física de tal modo que los alumnos construyan su propio aprendizaje.

\section{MATERIAL Y METODOS}

Materiales: En desuso y todo lo que se pudo reciclar, electrónicos: transistores, relays, bobinas, diodos, PC.

\section{Métodos:}

Fase I.- Se procedió inicialmente con la investigación bibliográfica. Se utilizó la recuperación de tecnologías a través de técnicos electricistas, electrónicos respecto a la electricidad sus aplicaciones y su relación con el magnetismo, entrevistas a físicos e investigadores.

Fase II.- Construcción de experimentos del "Demostrador Múltiple Experimental de Electricidad y Magnetismo", diseño y elaboracion del sistema electronico de control.

\section{RESULTADOS}

Se construyeron 14 experimentos de electricidad estática y en movimiento, su relación con el magnetismo, manipulados desde la computadora y organizados en una maleta portátil entre los que tenemos: El péndulo eléctrico; demostrador del espectro magnético y líneas de fuerza de un imán permanente, galvanoscopio, voltímetro escolar, bobina móvil, relay, reóstato, motores de corriente continua (DC) y corriente alterna (AC), transformador, válvula de seguridad, demostrador de obtención de calor y luz a través de la electricidad, generador de corriente por la acción de un campo magnético. Se elaboró un progra- ma en BORLAND C++ para decidir cual de las experiencias queremos observar, se puede leer en el monitor una breve explicación de la experiencia escogida, para ello se ingresa los datos mediante el teclado y al ser procesados envíen la orden a la experiencia elegida.

Los alumnos mostraron creatividad, curiosidad natural y una gran disposición por aprender investigando al construir, modificar, demostrar funcionando sus propios experimentos se motivaban frecuentemente por propia iniciativa, surgían preguntas las que se debatían en el aula, el profesor actuaba como orientador, dejando que ellos realicen todas las etapas de la investigación. El trabajo final "Demostrador Múltiple Experimental de Electricidad y Magnetismo" fue ganador nacional área ciencias básicas FENCYT 2002 y obtuvo el 2do lugar área electrotécnica 18 MOSTRATEC - Brasil 2003.

\section{DISCUSION}

En los textos escolares de nivel básico $(1,2,34)$ encontramos definiciones sobre el tema de estudio, es común en el sistema tradicional memorizarlos y limitarse a desarrollar los ejercicios propuestos previo al examen, al optar por priorizar que los alumnos recopilen información a través de sus fichas y elaboren sus conclusiones de grupo que fueron: a) En la electricidad estática los electrones no se mueven mientras que en la electricidad dinámica los electrones están constituidos por electrones móviles. b) El descubrimiento de Oersted permitió relacionar las propiedades eléctricas y magnéticas las cuales obedecen a las mismas leyes. c) La resistencia eléctrica de un material depende de 3 factores: de la longitud del conductor del grosor del conductor, del material que esta hecho el conductor. d) La resistencia es constante y en la ley de Ohm los materiales pueden ser buenos, malos o semi conductores. e) Algunos efectos que produce la electricidad son térmica, donde demostramos la ley de Joule otro efecto es la luminosa. f) La electricidad puede producir magnetismo y el magnetismo puede producir electricidad. Tampoco se descuidó la construcción de experimentos algunos de ellos propuestos en $(5,6,7)$, los que fueron adecuados a nuestros objetivos, algunos fueron modificados en forma y diseño por lo que se manifestó el análisis crítico y creatividad an- tes que la memoria y pasividad.

De la elaboración del informe final siguiendo pautas de investigación universales $(8,9)$ se desprende que los alumnos interiorizaron de manera práctica los contenidos del tema elegido, aprendieron haciendo de manera práctica, los múltiples experimentos construidos durante la investigación puede ser elaborados de forma sencilla y práctica por cualquier alumno, maestro o aficionado a la física con similares realidades socioeconómicas a la de los alumnos. Con lo expuesto y los halagadores resultados obtenidos se destierra la idea que la enseñanza de la física es difícil, aburrida, que la falta de materiales y la marginalidad no sean excusas del subdesarrollo científico y tecnológico. Y como consecuencia la manera diferente de aprender investigando en las escuelas secundarias del Perú es viable y pertinente por los resultados obtenidos, con la pertinente adecuación a cada realidad regional.

\section{AGRADECIMIENTOS}

Al doctor Raúl Ishiyama Cervantes y al doctor Manuel Respaldiza, a los alumnos, padres de familia y profesores del Colegio Estatal "San Luis", a Mercedes Quispe Ramos y Rocío Ayre Aire, a las empresas Crerecrok's y Kiwigen, al CONCYTEC. 


\section{REFERENCIAS BIBLIOGRÁFICAS}

1. Chunga Yaya, José. Física. Lima: Editorial Magisterio; 1996.

2. Maiztegui, Alberto P; Sabato, Jorge A. Introducción a la Física. Buenos Aires: Editorial Kapelus S.A.; 1978.

3. Santillana. Física. Lima: Ediciones Santillana S.A.; 1995.

4. $\quad$ Genzer, Irwin; Philip, Yougner. Física. México: Publicaciones Cultural S.A.; 1974.

5. Ávila Adolfo. Y Machío del Carmen. Tecnología. Madrid: McGraw Hill; 1996.

6. Graf F., Rudolf. Electricidad experimental para todos. Barcelona: Editorial Ramón Sopena S.A.; 1994.

7. Wood R. Física para niños. México: Metropolitana de Ediciones S.A. de C.V.; 1994.

8. Balarezo Gerstein, Naldo. Editor. Compilación de Normas y Criterios Para la Edición de Publicaciones Científicas. Lima: CONCYTEC; 2003.

9. Ishiyama Cervantes, Raúl. Pautas Para la Publicación de Artículos Científicos. [Publicación virtual] Lima: Universidad Peruana Cayetano Heredia; 1999. En: En: http://www.upch.edu.pe/upchvi/lnvestigación/pautas/ upch.html

E-mail: nabetse11@yahoo.es 